\title{
Lysosomal Deficiencies and Cell Death in Mucolipidosis Type IV
}

\author{
G. Colletti ${ }^{a}$, Y. Rbaibi ${ }^{a}$, M. Meidel ${ }^{b}$, J. Jennings ${ }^{a}$, O. A. Weisz ${ }^{b}$, and K. I. Kiselyov ${ }^{a}$ \\ ${ }^{a}$ Department of Biological Science, University of Pittsburgh, Pittsburgh, PA, 15260 USA; \\ e-mail: kiselyov+@pitt.edu \\ ${ }^{b}$ Renal and Electrolyte Division, University of Pittsburgh, Pittsburgh, PA, USA
}

DOI: $10.1134 / \mathrm{S} 1990747809030167$

Lysosomal storage diseases are a set of genetic diseases that occur due to dysfunction or mislocalization of proteins responsible for degradation of the endocytosed material. As a result, the cells accumulate storage bodies containing undigested proteins, lipids and other high-molecular compounds. A majority of storage diseases is associated with developmental delays, blindness and premature death. One of such diseases, mucolipidosis type IV (MLIV), is caused by mutation in a gene MCOLN1. MCOLN1 codes for a novel channel mucolipin 1 (TRPML1), which is localized in lysosomes. The two main models that attempt to explain the function of this ion channel suggest that it regulates: 1) membrane traffic within the lower portions of the endocytic pathway or 2) ionic conditions in the lysosomes and, therefore, the activity of lysosomal digestive enzymes. Identifying the role of TRPML1 in the endocytic pathway will define the direction of the future search for MLIV treatments.

The previous attempts to define the TRPML1 role were hampered by the use of MLIV patient cell cultures as model systems. The chronic accumulation of undigested lipids and proteins in such cells may affect the membrane traffic in the endocytic pathway and, therefore, manifest as delays in membrane traffic. In order to circumvent this issue, we used short interfering RNA that induced an acute decrease in cellular TRPML1 levels, and used this system to study membrane traffic. Our observations did not reveal any significant membrane traffic delays in the endocytic pathway of acutely TRPML1 deficient cells. These data show that MLIV is a "cellular metabolic disorder" and, therefore, the future MLIV treatments should probably focus on enzyme replacement therapies.

MLIV and other lysosomal storage disorders are neurodegenerative. Why lysosomal storage diseases induce cell death is unknown. We found that the suppressed lysosomal function in cells affected by MLIV impairs autophagy, which leads to the buildup of undigested aged dysfunctional mitochondria that can not protect cells from pro-apoptotic effects of cell stimulation. These results illuminate the cell death mechanisms of lysosomal storage diseases and suggest the use of apoptosis inhibitors as complimentary approaches to treating lysosomal storage diseases. 